\title{
INNOVACIÓN SOCIAL Y TECNOLÓGICA EN LA ACTIVIDAD ARTESANAl de MADERA EN la COMUNIDAd de DZITYÁ, YUCATÁN
}

\author{
SOCIAL AND TECHNOLOGICAL INNOVATION IN WOODCRAFT ACTIVITY IN THE DZITYÁ COMMUNITY, YUCATÁN
}

\author{
Ana Cristina Puc Cruz, José Francisco Sarmiento Franco, \\ Alfonso Munguia Gil \& Gustavo Monforte Méndez*
}

Tecnológico Nacional de México / Instituto Tecnológico de Mérida, México

*Corresponding author: anacristinapuc@ @otmail.com

\begin{abstract}
One the effects that globalization has brought, in addition to the socio-environmental impacts, is the promotion of technological innovation as a means to introduce new products and processes to the market and, in this way, promote economic growth. However, this growth does not consider its negative socio-economic implications, such as the increase in inequality, the loss of values, of the culture and ways of life of rural populations, and ecological, such as climate change and environmental deterioration. In this context, in the last decades the debate has arisen on the concept of social innovation that refers to new ideas, forms of social organization, new processes, new products, and new public policies that strengthen culture and help solve social and environmental issues from a sustainable development perspective. To this end, Higher Education Institutions play a very important role as promoters of knowledge and its application to solve the various problems that societies face, especially in the field of sustainability. The Dzityá Police Station in Yucatan has as its main source of income the elaboration of handicrafts of turned wood and stone carving, reason for which the artisans look for innovative alternatives in which they can market their products. For this reason, a group of local artisans and other state communities' under-take activities that contribute to the promotion of artisanal culture and fair trade in the locality.
\end{abstract}

Key words: Innovation, Technology, Culture, Crafts.

Resumen: Uno de los efectos que ha traído la globalización, además de los impactos socio-ambientales, es el fomento de la innovación tecnológica como un medio para introducir nuevos productos y procesos al mercado y, de este modo, fomentar el crecimiento económico. Sin embargo, este crecimiento no considera sus negativas implicaciones socioeconómicas, como el aumento de la desigualdad, la pérdida de valores, de la cultura y de los modos de vida de las poblaciones rurales, y ecológicas, como el cambio climático y el deterioro ambiental. En este contexto, en las últimas décadas ha surgido el debate sobre el concepto de innovación social que se refiere a las nuevas ideas, formas de organización social, nuevos procesos, nuevos productos, y nuevas políticas públicas que fortalezcan la cultura y contribuyen a resolver problemáticas sociales y ambientales, desde una perspectiva del desarrollo sostenible. Para ello, las Instituciones de Educación Superior, juegan un rol muy importante como promotoras del conocimiento y de su aplicación para la resolución de los diversos problemas que las sociedades enfrentan, especialmente en el ámbito de la sostenibilidad. La Comisaría de Dzityá en Yucatán tiene como principal fuente de ingresos económicos la elaboración de artesanías de madera torneada y tallado de piedra, motivo por el cual los artesanos buscan alternativas innovadoras en las cuales puedan comercializar sus productos. Por tal motivo, un grupo de artesanos locales y de otras comunidades del Estado emprenden la realización de actividades que contribuyen en la promoción de la cultura artesanal y el comercio justo en la localidad.

Palabras clave: Innovación, Tecnología, Cultura, Artesanías.

European Public \& Social Innovation Review

Volume 3, Issue 2, 2018 ISSN 2529-9824

Creative Commons 4.0. International 


\section{Introducción}

Al hacer referencia sobre las artesanías implica tener en mente una pluralidad de situaciones relacionadas con la producción y la comercialización vinculados con un conjunto de particularidades culturales, producto de determinadas condiciones históricas concretas que se vincula a factores tecnológicos, económicos, políticos, demográficos y simbólicos (Rotman, 2003).

El autor hace mención acerca de la elaboración de productos artesanales, la cual es continuamente apreciada como una expresión privilegiada de "lo local" que establece un interesante fenómeno económico-cultural desde donde pensar todos los aspectos que conllevan a la producción artesanal. Si bien, la elaboración de productos artesanales está ligada a diversos factores como lo menciona Rotman (2003) al principio; sin embargo, uno muy importante y que el autor no toma en cuenta es el que mencionan Domínguez, Hernández y Toledo (2004), que, para actualizar el sector artesanal, con vista a la actividad de la tecnología se debe fomentar la innovación en los procesos de producción artesanal.

Para las empresas artesanales debido a que se constituyen a través de las creencias y experiencias adquiridas en el pasado, las cuales en muchas ocasiones son bastante arraigadas les es difícil cambiar o aceptar lo nuevo. A pesar de ello hay empresas que tienen el deseo de salir adelante por lo que tratan de adaptarse a los cambios tecnológicos, buscando no afectar la manera tradicional en la cual se distingue a una artesanía.

Muchos artesanos prefieren seguir con las alternativas tradicionales que han sido parte de su familia por mucho tiempo, es por ello que cuando surge alguna tecnología nueva que pueda facilitar su producción, ventas, administración, y comercialización, primero analizan los beneficios que pueden obtener y la facilidad de adquirirla para después decidir si la adoptan.

En consideración a lo que se ha establecido y lo que se pretende abarcar en el presente trabajo debe quedar claro la función que tiene la tecnología en las empresas artesanales para que estás puedan sobrevivir a todos los cambios que se generan. De igual manera, la innovación social, así como la relación que pueda existir con los Institutos de Educación Superior (IES) juegan un papel importante pues a través de la innovación social los artesanos pueden generar conocimiento y cambiar el paradigma que tienen en cuanto al uso de las tecnologías; en lo que respecta a las IES son quienes en determinado momento pueden enseñar o crear tecnología, tal es el caso del Instituto Tecnológico de Mérida, pues realiza investigación y apoya a la Asociación de Artesanos de Madera Torneada de Dzityá A.C., en la búsqueda de soluciones a las problemáticas que enfrentan los artesa-nos de la comunidad.

\section{Consideraciones Teóricas}

\subsection{Conocimiento tradicional}

Los pueblos poseen conocimientos profundos llenos de las experiencias adquiridas de los antepasados y que se han transmitido de generación en generación. Estos saberes constituyen una parte indisoluble de su cultura, representan un valor estratégico para el desarrollo económico de los pueblos y contribuyen al desarrollo sostenible de las naciones (De la Cruz et al., 2005).

Adicionalmente los conocimientos, innovaciones y las prácticas tradicionales corresponden a los saberes con los que cuentan los pueblos indígenas los cuales se transmiten de generación en generación habitualmente de forma oral. Según De la Cruz et al., (2005), los saberes pueden ser tangibles e integrales a todos los conocimientos y practicas ancestrales por lo que constituyen la herencia intelectual colectiva de los pueblos indígenas. Sin embargo, muchos de esas sapiencias se van perdiendo con el paso del tiempo debido a que como dice el autor se transmiten oralmente, por lo tanto, no se lleva algún registro de ello.

En las palabras del autor los conocimientos tradicionales están relacionados con los siguientes saberes:

- Ciencias naturales (e.g. bióloga, botánica, zoología, taxonomía indígena).

- Lingüística, cantos, rituales, danzas y ritmos. 
- Curaciones, medicina y farmacóloga.

- Artesanía, cerámica, tejidos y diseños.

- Manejo de la biodiversidad, desarrollo sostenible, cultivos asociados, agroforestería, manejo de ecosistemas, manejo forestal y manejo de cuencas hidrográficas.

- Conocimiento de uso actual, previo o potencial de especies de plantas y de animales, así como de suelos y minerales, conocido por un grupo cultural.

- Conocimiento de preparación, proceso y almacenamiento de especies útiles.

- Conocimientos sobre conservación de ecosistemas.

- Ceremonias y curaciones realizadas dentro y fuera de su ámbito cultural.

- Sistemas de derecho consuetudinario y valores morales.

$\mathrm{Si}$ bien los saberes tradicionales se relacionan con una serie de conocimientos que pueden definir como un cuerpo acumulativo de conocimientos, prácticas y creencias que van cambiando a través de procesos de adaptación y que se transmiten mediante formas culturales de una generación a otra (Burrola et al., 2012 y Luna-Morales, 2002).

Al igual que el conocimiento científico, el conocimiento tradicional es el resultado de un proceso acumulativo y enérgico de experiencias, prácticas y adaptación al cambio. Un aspecto que distingue al conocimiento científico del conocimiento tradicional es que el segundo es local, holístico y portador de una cosmovisión que integra aspectos físicos y espirituales (Reyes-García, 2009). Por tal motivo este tipo de conocimiento es importante para las comunidades indígenas porque son parte de su identidad cultural, además representa la herencia de nuestros antecesores y refleja la gran diversidad de la humanidad.

Por otro lado, las artesanías se entienden como expresiones por medio de las cuales se manifiestan, representan y modelan identidades relacionadas con la nacionalidad, se basan y remiten a tradiciones, las cuales forman parte de una identidad cultural que se encuentra vinculada a una sociedad y a un estado determinado, además son relacionadas con ideas y valores que se asocian a un pasado centrado en términos de historia regional y nacional. Es interesante señalar que los bienes artesanales tienen la condición de ser propios de su realidad, la coexistencia de pluralidad (Rotman, 2003).

El autor señala que las artesanías forman parte del legado cultural del cada país, desde sus concreciones materiales e imagen plástica hasta en los aspectos que realizan en sus procesos productivos (técnicas, etc.) y en sus implicaciones simbólicas, identitarias e ideológicas. Debido a ello se incluyen entre los símbolos que definen a una nación.

Frecuentemente la gente suele asocial a las artesanías con una forma de producción ancestral, antigua, preindustrial, subdesarrollada, rural, originaria de las sociedades pobres, sin embargo, para Malo (2008) necesariamente no es como se plantea. Además, con el transcurso del tiempo esto ha cambiado tal vez se den cambios lentos o parciales; pero existen. El autor menciona como ejemplo que, en la antigua fragua de los herreros, avivada manualmente con un fuelle hecho de piel ha sido desplazada por el venterol, un artefacto movido por energía eléctrica. La quema de los productos cerámicos se hace generalmente en hornos eléctricos. El tradicional torno de pie de los alfareros puede ser reemplazado por un torno movido por un motor eléctrico.

Con base en lo anterior el autor comenta que las artesanías requieren de arte-sanos que las trabajen con métodos diferentes a la industria y que, en la mayoría de los casos, obtienen de la producción de los bienes artesanales los medios económicos para poder solucionar los problemas que se les presente, por lo tanto, implica diversas formas de comercialización que requiere en ocasiones del uso de tecnologías.

Entonces, las artesanías sufren cambios tecnológicos para que puedan subsistir e innovar en sus procesos, así como en sus productos. Por lo tanto, es imprescindible que los artesanos se encuentren preparados para las nuevas tecnologías que surjan y que se puedan adaptar a su actividad económica.

\subsection{Tecnología e innovación rural}

La búsqueda de un desarrollo para los territorios capaz de hacer compatible la competitividad de 
sus empresas con el mantenimiento de su población, así como mayores cuotas de bienestar, sustentabilidad ambiental y respeto por el patrimonio cultural heredado, esto constituye un reto para actores sociales y responsables públicos desde hace décadas. En ese contexto, identificar las estrategias que sean las más adecuadas para promover la dinámica de las regiones atrasadas, las ciudades pequeñas y las áreas rurales, junto con las actividades tradicionales y las pequeñas empresas (como los talleres artesanales), forma una línea de investigación de interés teórico y operativo que exige la colaboración de diferentes perspectivas profesionales (Méndez, 2006).

Por otra parte, Hernández et al., (2009) afirma que la sostenibilidad de lo artesanal supone el ingreso a dinámicas de mercado global de forma competitiva e innovadora por lo tanto exige de los artesanos patrones productivos que consideran los elementos clave: artefactos (artesanías), estos surgen de una memoria cultural, una tecnología la cual es representada en la acumulación de un conocimiento tradicional aplicado a un objeto y los recursos naturales que garanticen la duración de un capital natural, un factor importante para la permanencia de los productos artesanales tradicionales.

Actualmente aún podemos encontrar que hay personas que continúan viviendo como lo hacían sus antepasados, sin tener contacto con las máquinas y modificando muy poco su economía. Según Turok (1988, 22-194) en México aproximadamente sólo el 5\% del total de los artesanos han innovado y tienen éxito en esa actividad; pero existe un $65 \%$ de ellos que emplea sistemas rudimentarios y sobrevive de ese oficio. El otro $30 \%$ está en el punto medio. Varios autores consideran a la artesanía como una actividad complementaria a la agricultura de temporal, (Jiménez, 1982, 46-53; Bonfil, 2001, 9-490; Cook, 1995, 38; Turok, 1988, 22 194). Es por ello que hacen referencia del campesino pues él además de cultivar elabora productos no agrícolas; su forma de producción se tipifica como una organización o unidad doméstica familiar, porque cada miembro aporta conocimientos, habilidades, capacidades y pretende conservar su producción tradicional, de generación en generación. Sin embargo, ese modo del trabajo implica una división a varios niveles, que varía en grado de especialización: por sexos, grupos de edad y por actividad económica. Es así, como algunos procesos de producción se hicieron exclusivamente masculinos, otros, exclusivamente femeninos; otros más, mixtos. Alguna división del trabajo llega a otorgar a personas Otros tienen un gran número de tareas $o$ fases, o incorporan a sectores que generalmente no se consideran productivos, como el infantil y el de los ancianos. Este comportamiento del sector genera una tipificación propia de los factores de innovación.

Para señalar factores que intervienen en la producción artesanal se puede utilizar el trabajo de Jiménez (1982, 46-53) quien realizó un estudio en los altos de Chiapas, en el cual obtuvo como resultado que entre la población artesanal hay nueve familias que tienen ciertas prácticas que les han permitido posicionarse como líderes en el ramo, estás prácticas consisten en:

- Innovación con nuevos productos.

- Innovación introduciendo algunos elementos novedosos.

- Realizan pedidos al mayoreo a comerciantes de mercados lejanos.

- Innovación introduciendo diseños, moldes y modelos.

- Contratación de personal como fuerza de trabajo asalariada.

- Hay una construcción anexa a la casahabitación, para producir o para vender.

- Hay un almacén para los productos.

- Venden directamente a casas y almacenes de productos artesanales en otras localidades.

- Realizan viajes a las ciudades para "checar" precios.

- Tienen amplia información sobre precios y las fluctuaciones de oferta y demanda de los productos.

- Innovación imitando técnicas y estrategias de producción y comercialización de otras localidades.

- Viajan a otras localidades en busca de mejores mercados para su artesanía.

- Producen para instituciones con programas y proyectos para el desarrollo de las artesanías como: FONART (Fondo Nacional para el Fomento de las Artesanías), FONAFE 
(Fondo Nacional para el Fomento Ejidal).

- Tienen contacto con centros urbanos de mayor importancia que su localidad.

El autor como bien muestra en la lista de factores que intervienen en la producción de artesanías está involucrada la innovación en los productos artesanales, la búsqueda de nuevas formas de comercialización, así como posicionamiento de puntos estratégicos para la venta de los productos, por otro lado hace falta la contribución de la tecnología entre su planteamiento pues es un factor sumamente prescindible ya que, como sea mencionado anteriormente, los artesanos se encuentran inmersos en cambios tecnológicos que si no saben cómo adaptarse podría ocasionar que no se posicionen sus productos en el mercado.

\subsection{Innovación social en economías rurales}

Los conceptos de innovación social han cambiado y toman cada vez más relevancia entre un mundo de industrialización, tecnologías y producción, y otro de sostenibilidad y desarrollo de economías micro fortalecidas a través de la potencialidad de recursos locales con la intervención de comunidades y organizaciones que consoliden procesos de región.

Por lo tanto, la innovación social puede ser definida como "el desarrollo e implementación de nuevas ideas (productos, servicios y modelos) para satisfacer las necesidades sociales, crear nuevas relaciones sociales y ofrecer mejores resulta-dos. Sirve de respuesta a las demandas sociales que afectan al proceso de inter-acción social, dirigiéndose a mejorar el bienestar humano" (European Commsion s.f. pp. 4). Es una nueva forma de innovar. Se puede decir que esta nueva manera de crear innovación se centra en las relaciones sociales pues como lo dice en la definición satisface las necesidades sociales y para lograrlo es necesario entablar relaciones sociales con el fin de conocer los problemas de las personas más necesitadas para poder generar soluciones.

Por otro lado, este concepto ha despertado en los últimos años un interés, debido a que tiene que ver con las complejas problemáticas que enfrenta el mundo a nivel global en temas que hoy en día se han vuelto tan comunes como: la salud, educación, el medio ambiente, entre otros. Estos temas que interesa a la innovación social son temas globales, sin embargo no implica que las soluciones puedan ser iguales para todas las comunidades en la que se genere esta nueva forma de innovar, sin embargo, puede ser que mediante las ideas que surjan para la solución de problemas se puedan hacer combinaciones que puedan ser replicadas de un lugar a otro con adaptaciones a sus distintos entornos en aspectos cultura-les y económicos, donde los métodos tradicionales ya no generan soluciones y necesitan ser corregidos con eficiencia.

Una de las organizaciones más importante a nivel mundial es la Comisión Económica para América Latina y el Caribe (CEPAL), la cual viene trabajando desde el año 2004 en el tema de innovación social. Esta organización ha encontrado innumerables ejemplos sobre el tema, dado que los estados no le dan res-puesta a todas las necesidades de los individuos que los conforman CEPAL (2015). En el mismo contexto este organismo menciona que la innovación social se da como respuesta de la sociedad civil, las comunidades y al mismo gobierno ya que ya no se pueden resolver los problemas con los procesos o métodos tradicionales.

A través del tiempo se han dado cuenta que actualmente ya son más evidentes y acentuadas las problemáticas de las comunidades que suelen ser vulnerables por lo tanto es necesario otorgar respuestas efectivas y novedosas a sus necesidades, aunque en ocasiones parezcan básicas. Otra definición interesante sobre innovación social es la que hace la CEPAL (2015) pues define este término como aquella que se caracteriza por desarrollar nuevas maneras de administrar y ejecutar; lo cual involucra el uso de nuevas herramientas, medios y combinación de factores, encaminados a alcanzar una mejoría de las condiciones sociales y de vida en general de la población de la región.

Otros autores como Phills, Deiglmeier y Miller (2008), coinciden en que es una solución a un problema social que es más eficaz, eficiente y sostenible que las soluciones existentes cuyo valor creado se acumula en la sociedad en su conjunto $y$ no en las 
particularidades (citado en León, Baptista \& Contreras, 2012). Es importante mencionar que la innovación solo se puede considerar social, si responde a mejorar el bienestar de la sociedad y que no fomente la riqueza de un grupo u organización específica, sin importar que este tipo de innovación se dé en nivel de productos o procesos, lo que debe ser trascendental es el cambio que pueda originar en la sociedad en la cual esté presente en las relaciones la innovación social (Camargo et al., 2017).

\section{Metodología}

Para la elaboración del presente artículo y poder adquirir información relevante sobre el tema se realizó una revisión documental, la cual es necesaria para comprender los temas mencionados en el trabajo. De manera que se pueda compren-der los temas no solo de manera teórica, sino que también práctica.

La presente investigación se enfocará en el sector artesanal de madera de Dzityá, Yucatán. Una comisaría localizada a 15 kilómetros al norte del centro de la ciudad de Mérida, capital del estado de Yucatán, cuenta con 1,260 habitantes (INEGI, 2010). Debido al crecimiento urbano que se ha generado en la periferia de la comunidad se le considera ya un suburbio de la ciudad capital del estado, además es conocida regionalmente por la elaboración de artesanías de tallado de madera torneada y por el labrado de piedra de cantera.

El estudio principalmente se realizó con los integrantes (seis artesanos) de la Asociación de artesanos de Madera Torneada de Dzityá A.C., ya que son un grupo establecido, además que los integrantes cuentan con talleres propios y forma-les, otro aspecto que fue relevante para la realización de la investigación es que el Instituto Tecnológico de Mérida lleva cuatro años trabajando con ellos y cuentan con un convenio firmado.

La información requerida para el trabajo fue realizada por medio de la Investigación Acción Participativa (IAP) ya que por medio de esta metodología se lograría la participación e inclusión de los artesanos en la búsqueda de soluciones a los problemas que tienen relacionados principalmente con la innovación y la tecnología, para ello se realizó un diagnóstico (Colmenares, 2012), el cual se realizó por medio de entrevistas individuales, además de la observación participante, esto se realizó debido a que entre los actores estudiados no hay colectividad a pesar de ser un grupo formado (Durston \& Miranda , 2002).

Otro aspecto importante de esta metodología es que está dirigida a los problemas comunitarios, además que la (IAP) genera los medios para que las personas involucradas recuperen su capacidad de pensar por sí misma, de igual manera forja las capacidades necesarias para desarrollar innovación social en el entorno artesanal, además contribuye a la conservación de la cultura para la preservación de una vida autentica (Lewin et al., 1990).

Por último, con la IAP se logra construir las acciones que se deben tomar para la solución de los problemas, también contribuye en el análisis y reflexión del esta-do actual de los artesanos en cuanto a la innovación y tecnología con la que cuentan (Colmenares, 2012).

\section{Resultados}

En base a lo que menciona De la Cruz et al., (2005) los artesanos de la comunidad de Dzityá cuentan con los saberes adquiridos de sus antepasados, los cuales se han transmitido de generación en generación, es una característica importante pues los talleres artesanales son familiares. La técnica artesanal constituye una parte indisoluble de la cultura de esta localidad, además representa un valor estratégico que contribuye en el desarrollo económico y al desarrollo sostenible (De la Cruz et al., 2005).

En total se encontró que los seis artesanos estudiados de la comunidad de Dzityá, Yucatán, también realizan actividades similares a las que menciona Jiménez (1982). Ellos se organizan para comercializar sus productos artesanales por medio de un encuentro regional de artesanos, lo cual realizan cada mes, además que han buscado con ayuda del Instituto Tecnológico de Mérida apoyo de algunas dependencias gubernamentales como es el caso de Fomento Económico y Turístico del municipio de Mérida, Yucatán cuyo apoyo consiste en brindar el mobiliario necesario para poder llevar a cabo los eventos. Asimismo, se ha obtenido la colaboración de Fomento Cultural, cuyo aporte ha sido de proveer con eventos culturales para llamar la atención de la gente y de esta manera 
atraerlas al poblado para consumir los productos que se elaboran en la comunidad. Así como esta actividad, también participan en ferias artesanales o de comercio organizados por organizaciones como la Cámara Nacional de Comercio (CANACO), la casa de artesanías, el H. Ayuntamiento del Municipio de Mérida, el CDI, entre otras. Otro aspecto, es que están en la búsqueda de proveedores que les ofrezcan madera certificada, esto para evitarse problemas con la CONAFOR, además que apoyan en la conservación de especies.

En cuanto a la tecnología, se obtuvo como resultado que los artesanos utilizan las redes sociales para la venta de sus productos, así como para difundir el evento artesanal que realizan periódicamente. Solamente un artesano cuenta con una página WEB la cual utiliza para darse a conocer nacional e internacionalmente, así como para buscar alguna capacitación que le sea de utilidad para desarrollar mayores habilidades que contribuyan en hacer crecer su taller artesanal. Cabe mencionar que es una mujer la dueña de la pequeña empresa que cuenta con el sitio WEB, además que los productos que vende son realmente innovadores pues hace accesorios de madera (collares, pulseras, aretes, etc.), es la única mujer que pertenece a la Asociación y la única en la comunidad que realiza este tipo de trabajo. Otro aspecto relacionado la tecnología es la maquinaria que utilizan los artesanos para la elaboración de sus productos, como ha mencionado Malo (2008) con el transcurso del tiempo esto ha cambiado, pues ahora las maquinas son más modernas, en el caso de esta técnica los antepasados utilizaban tornos de madera bastante rústicos. En la actualidad los tornos son eléctricos, modernos y los artesanos pueden adaptarlos a sus necesidades con la ayuda de un soldador de la comunidad, también acuden con el soldador para construir maquinas que se adapten a sus necesidades, como es el caso de un artesano que adapto una sierra eléctrica a una base con la finalidad de facilitar el corte de la madera, ya que en ocasiones compran troncos grandes y es complicado cortarlos.

También se obtuvo que algunas instituciones educativas contribuyen para que los artesanos tengan tecnología que les optimice el proceso de producción, como es el caso de la escuela "Modelo" de Mérida que crearon una estufa para el seca-do de la madera que funciona con un panel solar, sin embargo, el proceso sigue siendo tardado. Otra institución educativa que contribuye con los productores artesanales es el Instituto Tecnológico de Mérida, pues en algún momento realizó una capacitación de computación para los artesanos ya que ellos no sabían cómo se utiliza una computadora, sin embargo, no fue suficiente por lo que aún requieren de este tipo de actividad, así como se les dio esta capacitación se realizó con ellos una serie de cursos relacionados con la administración, costos y de seguridad e higiene. Se obtuvo la colaboración con la Universidad HEC de Montreal, Canadá en la elaboración de la página de Internet y redes sociales de la Asociación de Artesanos de Madera Torneada de Dzityá A.C. con esto han lo-grado demostrar actualización en sus acciones, a pesar de contar con esta herramienta, los artesanos desconocen cómo utilizar la página WEB, por lo tanto, aun les hace falta trabajar en este aspecto para que puedan aprovechar esta oportunidad de innovación y tecnología con la que cuentan. Por lo tanto, se puede encontrar que intervienen diferentes actores en el desarrollo de las empresas artesanales (Méndez, 2006).

A pesar de las complicaciones que puedan tener los participantes aún está presente en ellos el interés de hacer acciones innovadoras que les ayude mejorar sus procesos de producción así como la comercialización de sus bienes artesanales, y saben que para ellos es necesario adaptarse a las tecnologías que surjan a través del tiempo, es por ello que ya están tomando las medidas necesarias para lograrlo, pues tres de los que no cuentan con el sitio WEB, ya están haciendo lo necesario para poder crearlo y de esta manera obtener mayores posibilidades de comercialización, además que involucran a su familia, ya que las personas que se encargaran de alimentar la herramienta serán los hijos que cuentan con estudios universitarios.

Por último, la innovación social ha estado presente entre la comunidad artesanal pues se han identificado acciones que la generan como es la generación de nuevas ideas de comercialización, de organización en la realización de los eventos culturales, así como en los propios talleres familiares ya que desde siempre buscan el bienestar de los integrantes de la familia, por otro lado, se encuentra la 
Asociación que busca el bienestar de la comunidad artesanal (European Commision s.f. pp. 4). Otro aspecto, que contribuye en la innovación social es la relación que se ha entablado con las IES y las organizaciones gubernamentales pues entre este triangulo (artesanos, IES y gobierno) se puede generar innovar en las relaciones sociales con la finalidad de generar soluciones.

\section{Discusión y reflexiones finales}

Como bien se ha mencionado es importante continuar construyendo vínculos con las IES pues fomenta la innovación, contribuyen a la solución de problemas de los artesanos de madera, favorecen el aprendizaje entre los actores, además ayudan en la búsqueda de mejoras de los procesos con los que cuenta la población estudiada.

Por otro lado, se encuentra el conocimiento científico y el conocimiento tradicional que son producto de un proceso acumulativo y dinámico de experiencias prácticas y adaptación al cambio. Una diferencia que existe entre dichos conocimientos es que el tradicional es local, holístico y portador de una cosmovisión que vincula aspectos físicos y espirituales. Sin embargo, la comunidad artesanal trata de vincular estos de manera que sean utilizados en la solución de sus problemas pues ambos conocimientos son importantes para la existencia de la técnica artesanal.

Por lo anterior, se requiere de estrategias para generar tecnología que sea de utilidad a los artesanos, que conserve la esencia cultural con la que cuenta este tipo de producción, así mismo de generar cohesión entre la comunidad artesanal para que de esta manera los proyectos que surjan se realicen mediante la colaboración de los actores involucrados y se logre una verdadera colectividad en la Asociación.

Por último, es importante que las Instituciones de Educación Superior (IES) generen tecnología propia para los artesanos, que los involucren en los procesos, con la finalidad de generar conocimiento entre los actores, así como una transferencia del mismo que pueda ser utilizado por todos los involucrados. 


\section{Referencias}

Burrola-Aguilar, C., Montiel, O., Garibay-Orijel, R., and Zizumbo-Villarreal, L. (2012). Conocimiento tradicional y aprovechamiento de los hongos comestibles silvestres en la región de Amanalco, Estado de México. Revista mexicana de micología, 35, 01-16.

Camargo, J. E. P., Contreras, F. G., and Jiménez, Y. Y. R. (2017). Estado del arte de la innovación social: una mirada a la perspectiva de Europa y Latinoamérica. Opción: Revista de Ciencias Humanas y Sociales, 82, 563-587.

Colmenares, A. (2012). Investigación-acción participativa: una metodología integradora del conocimiento y la acción. Voces y Silencios: Revista Latinoamericana de Educación, 102-115.

De la Cruz, R., Muyuy Jacanamejoy, G., Viteri Gualinga, A., Flores, G., Humpre, J. G., Mirabal Díaz, J. G., and Guimaraez, R. (2005). Elementos para la protección sui generis de los conocimientos tradicionales colectivos e integrales desde la perspectiva indígena. CAF, 43.

Domínguez, H. M., Hernández, G. J., and Toledo, L. A. (2004). Competitividad y ambiente en sectores fragmentados. El caso de la artesanía en México. Cuadernos de Administración, 17 (27), 127-158.

Durston , J., and Miranda , F. (2002). Experiencias y metodología de la investigación participativa. CEPAL, SERIE Políticas sociales, 5-71.

Hernández., de la Paz, J., Domínguez Hernández, M. L., and Caballero, M. (2009). Factores de innovación en negocios de artesanía de México. Gestión y política pública, 16(2), 353-379.

INEGI. (2010). Censo de Población y Vivienda. Obtenido de INEGI: www.inegi.org.mx

Jiménez, M. (1982). "Huáncito la alfarería en una comunidad purépecha", Ensayos 7, México, UAMAzcapotzalco.

Luna-Morales. (2002). Ciencia, conocimiento tradicional y etnobotánica. Etnobiología, 2, 121-135.

Lewin, K., and Salazar, M. C. (1992). La investigación-acción participativa: inicios y desarrollos. Editorial Popular.

Malo, C. (2008). Artesanías, lo útil y lo bello. Cuenca: Cidap, Universidad del Azuay.

Méndez, R. (2006). La construcción de redes locales y los procesos. Problemas del desarrollo, 218-240.

Reyes-García. (2009). Conocimiento ecológico tradicional para la conservación: dinámicas y conflictos. Papeles, 39-55.

Rotman, M. (2003). Modalidades productivas artesanales: expresiones de "lo local" en un mundo globalizado. Artigos, 135-145. 